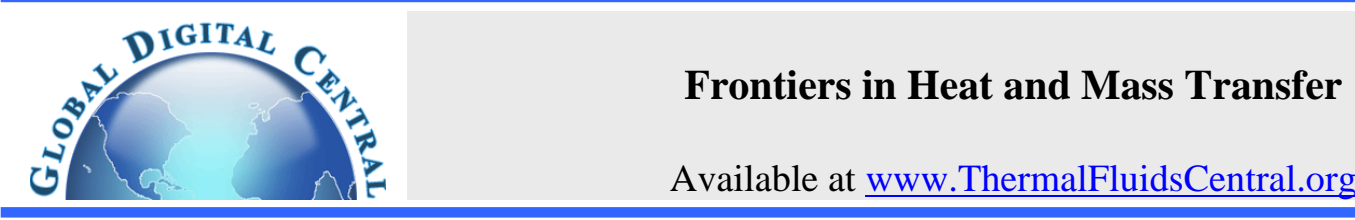

\title{
PERISTALTIC FLOW OF CASSON LIQUID IN AN INCLINED POROUS TUBE WITH CONVECTIVE BOUNDARY CONDITIONS AND VARIABLE LIQUID PROPERTIES
}

\author{
C. Rajashekhar ${ }^{\mathrm{a}}$, G. Manjunatha ${ }^{\mathrm{a}, \dagger}$, Hanumesh Vaidya $^{\mathrm{b}}$, B. B. Divya ${ }^{\mathrm{a}}$, K. V. Prasad ${ }^{\mathrm{c}}$ \\ a Department of Mathematics, Manipal Institute of Technology, Manipal Academy of Higher Education, Manipal, Karnataka, 576104, India \\ ${ }^{\mathrm{b}}$ Department of Mathematics, SSA Government First Grade College (Autonomous), Ballari, Karnataka, 583101, India \\ ${ }^{\mathrm{c}}$ Department of Mathematics, Vijayanagara Srikrishnadevaraya University, Ballari, Karnataka, 583105, India
}

\begin{abstract}
The primary objective of this paper is to examine the impact of variable viscosity and thermal conductivity on peristaltic transport of Casson liquid in a convectively heated inclined porous tube. The viscosity differs over the radial axis, and temperature dependent thermal conductivity is taken into account. The perturbation technique is utilized to solve the governing nonlinear equations under the assumption of long wavelength and small Reynolds number. The analytical solutions are obtained for velocity, streamlines, pressure rise, frictional force, and temperature when subjected to slip and convective boundary conditions. The impacts of related parameters on physiological quantities of interest are discussed and analyzed through graphs. It is seen that the variable viscosity has a noteworthy part in upgrading the velocity profiles. The investigation additionally demonstrates that the size of trapped bolus diminishes with an expansion in the velocity slip parameter.
\end{abstract}

Keywords: Darcy number; thermal conductivity; variable viscosity; velocity slip.

\section{INTRODUCTION}

Peristaltic mechanism of Newtonian/non-Newtonian liquids assumes a critical part in understanding the physiological behavior of various biological fluids. Specifically, in the movement of blood flow in small arteries and the chyme transport in the gastrointestinal tract helps the biomedical engineers to design and construct dialysis and heart-lung machines. The mechanism of peristalsis can be found in various regular frameworks, for instance, bolus transport through the esophagus, the advancement of spermatozoa in the cervical channel of the male reproductive tract and the stream of urine through the ureter. The early literature on peristaltic stream was carried out by taking Newtonian fluid with different supposition and geometries (Shapiro et al. (1969)). But most of the liquids occurring in nature are non-Newtonian, where there exists a nonlinear relation between stress and strain. Mainly, a significant portion of the biological fluids like blood and chyme transport in gastrointestinal tracts fall under the non-Newtonian classifications. Thus, it is necessary to analyze the flow behavior of these liquids by considering the non-Newtonian fluid models. Keeping this in mind, Raju and Devanathan (1972) investigated the non-Newtonian behavior of peristaltic transport by using the power law model. Vajravelu et al. (2005) analyzed the peristaltic transport of Herschel-Bulkley fluid in a slanted tube and compared the results for Newtonian, Power-law and Bingham-Plastic models. Recently, Rajashekhar et al. (2018) investigated the peristaltic transport of two-layered blood flow in an axisymmetric tube.

The impacts of heat transfer on classical and biological fluids play a significant role in nuclear plants, bioengineering devices and have different applications in industries. The heat transfer in most of the systems can occur in three modes; convection, conduction, and radiation. Specifically, the convective way of heat transfer plays an essential role in understanding the heat exchange between many biological tissues. Additionally, the impact of porosity along with heat transfer has been of vital importance because of its application in understanding the different mechanism in lungs, gallbladder, movement of blood flow through narrow arteries, and so on. In the human body, a substantial part of the muscle is subjected to porous structures. These structures are necessary to supply the supplements to each cell, and their proper working fundamentally relies on the blood coursing through them. In such circumstances, the nearness of slip on the boundary because of the porosity of the wall has an essential influence in examining the flow of blood in arteries. Motivated by the applications of porosity along with the convective conditions at the walls, Akbar (2014) analyzed the peristaltic transport of a nanofluid flowing through porous channels with convective boundary conditions. Recently, several researchers have examined the heat transfer characteristics on classical and biological fluids in different geometries and configurations (Nadeem and Akbar (2009); Ramesh (2016); Hayat et al. (2016); Mebarek-Oudina and Bessaih (2016); Kumar and Abzal (2017); Wakif et al. (2018a,b,c);

${ }^{\dagger}$ Corresponding author. Email: gudekote_m@rediffmail.com 
Sankad and Patil (2018)).

Among the several non-Newtonian models (Hayat et al. (2018a,b,c); Vaidya et al. (2018)) the Casson model is more appropriate for understanding the complex rheological behavior of blood. Srivastava and Srivastava (1984) investigated the peristaltic transport by utilizing the Casson model for the flow of blood. Mernone et al. (2002) examined the two-layered peristaltic transport by employing the Casson model and inferred that the Casson model could be utilized for investigating the physiological behavior of blood in small arteries and urine flow through the ureter. Ramesh and Devakar (2015) examined the impact of slip velocity on peristaltic transport of Casson fluid. Recently, various researchers have utilized the Casson model to understand the complex behavior of many classical and biological fluids in different geometries and configuration (Khalid et al. (2015); Vajravelu et al. (2017); Manjunatha and Rajashekhar (2018); Khan et al. (2018); Zia et al. (2018)).

The more significant part of the above examinations has investigated the Newtonian/non-Newtonian characteristics of the fluid flow by taking constant thermophysical properties of the fluid. These properties may change regarding temperature variation, especially the thermal conductivity. Therefore, it is essential to consider these properties to explore the peristaltic transport of biological fluids in various geometries and configurations. Thus, considering variable liquid properties will give rise to the broader understanding of classical and biological fluids (Hayat et al. (2014); Hussain et al. (2015); Prasad et al. (2017a,b)). Along these lines, the supposition of constant viscosity fails to clear up the peristaltic mechanism involved in blood flow, lymphatic vessels and bolus transport in the esophagus. In these organs, the viscosity of the fluid contrasts over the thickness of the channel/tube (Hayat and Ali (2008); Sinha et al. (2015); Bhatti and Zeeshan (2016)).

Inspired by the examinations carried out by different researchers, the endeavor has been made to investigate the peristaltic transport of Casson liquid under the influence of variable viscosity, thermal conductivity and convective boundary conditions in an inclined porous tube. The governing nonlinear equations are solved by using perturbation technique subjected to slip and convective boundary conditions. The analytical solutions are obtained for velocity, streamlines, flow rate, pressure rise, frictional force, and temperature. Furthermore, the influences of appropriate parameters on physiological quantities of interest are analyzed and discussed graphically.

\section{FORMULATION OF THE PROBLEM}

Consider the peristaltic transport of incompressible viscous liquid in an inclined tube of radius $a$ (Fig. 1). Casson model is used to represents the non-Newtonian behavior of the fluid. The flow happens through an axisymmetric porous tube and encourages the decision of fixed frame $(R, \Theta, Z)$ to study the problem. Let $u$ and $w$ be the radial and axial velocity components respectively. In the region between $r=0$ and $r=$ $r_{p}$ (plug flow region), we have $\tau_{r z} \leq \tau_{0}$. The region between $r=$ $r_{p}$ and $r=H$ (core region), we have considered $\tau_{r z} \geq \tau_{0}$. The wall deformation due to a sinusoidal wave trains traveling with the wave speed $c$ along the walls of a distensible tube is given by (Nadeem and Akbar (2009))

$$
H(z, t)=a+b \sin \left[\frac{2 \pi}{\lambda}(z-c t)\right]
$$

where $b$ is the amplitude, $z$ is the axial coordinate, $\lambda$ is the wavelength and $t$ is the time.

The flow turns out to be steady in the wave frame $(r, \theta, z)$ moving with velocity $c$ from the fixed frame $(R, \Theta, Z)$ given by (Nadeem and Akbar (2009))

$$
r=R, z=Z-c t, \psi=\Psi-\frac{R^{2}}{2}, p(Z, t)=P(z), \theta=\Theta
$$

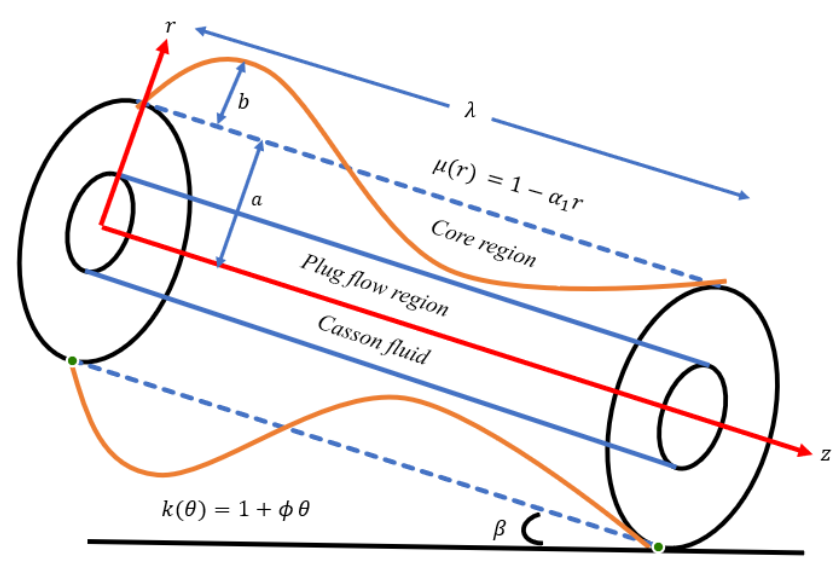

Fig. 1 Geometry of a peristaltic transport through an inclined axisymmetric porous tube.

where $\psi$ and $\Psi$ are stream functions, $p$ and $P$ are pressures, in wave and fixed frames of references, respectively.

The expressions for variable thermal conductivity and variable viscosity are given by (Hayat et al. (2014); Sinha et al. (2015))

$$
k(\theta)=1+\phi \theta, \quad \mu(r)=1-\alpha_{1} r \text { for } \phi<<1 \text { and } \alpha_{1}<<1
$$

where $\phi$ and $\alpha_{1}$ are the coefficients of variable thermal conductivity and variable viscosity respectively.

To non-dimensionalize the mathematical formulation, we define the following non-dimensional quantities

$$
\begin{aligned}
& \bar{r}=\frac{r}{a}, \bar{z}=\frac{z}{\lambda}, \bar{t}=\frac{c t}{\lambda}, \overline{\tau_{0}}=\frac{\tau_{0} a}{\mu_{0} c}, \tau_{r z}^{-}=\frac{\tau_{r z} a}{\mu_{0} c}, \bar{p}=\frac{p a^{2}}{\lambda c \mu_{0}}, \overline{r_{p}}=\frac{r_{p}}{c}, \\
& \epsilon=\frac{b}{a}, F_{1}=\frac{\mu_{0} c}{\rho g a^{2}}, \operatorname{Re}=\frac{\rho c a}{\mu_{0}}, \operatorname{Pr}=\frac{\mu_{0} c_{p}}{k}, \theta=\frac{T-T_{0}}{T_{0}}, \bar{w}=\frac{w}{c}, \\
& \bar{u}=\frac{u}{c}, E c=\frac{c^{2}}{c_{p} T_{0}}, \delta=\frac{a}{\lambda}, \overline{\tau_{a}}=\frac{\tau_{a} a}{\mu_{0} c}, \mu \overline{(r)}=\frac{\mu(r)}{\mu_{0}}
\end{aligned}
$$

The non-dimensional equations of motion and energy in the wave frame of reference, moving with velocity $c$, under the lubrication approach is as follows (Nadeem and Akbar (2009)):

$$
\begin{aligned}
& \operatorname{Re} \delta\left(u \frac{\partial}{\partial r}+w \frac{\partial}{\partial z}\right) w=-\frac{\partial p}{\partial z}+\frac{1}{r} \frac{\partial}{\partial r}\left(\tau_{r z}\right)+\delta \frac{\partial}{\partial r}\left(\tau_{z z}\right) \\
& \operatorname{Re} \delta^{3}\left(u \frac{\partial}{\partial r}+w \frac{\partial}{\partial z}\right) u=-\frac{\partial p}{\partial r}+\frac{\delta}{r} \frac{\partial}{\partial r}\left(\tau_{r r}\right)+\delta^{2} \frac{\partial}{\partial r}\left(\tau_{r z}\right) \\
& \operatorname{Re} \delta \operatorname{Pr}\left(u \frac{\partial}{\partial r}+w \frac{\partial}{\partial z}\right) \theta=B r\left(\delta \frac{\partial u}{\partial r} \tau_{r r}+\frac{\partial w}{\partial r} \tau_{r z}+\delta^{2} \frac{\partial u}{\partial r} \tau_{z r}\right. \\
& \left.+\tau_{z z} \frac{\partial w}{\partial r} \delta\right)+\frac{\partial^{2} \theta}{\partial r^{2}}+\frac{1}{r} \frac{\partial \theta}{\partial r}+\delta^{2} \frac{\partial^{2} \theta}{\partial z^{2}}
\end{aligned}
$$

where $u$ and $w$ are the radial and axial velocities, Re is the Reynolds number, $\theta$ is the temperature, $\delta$ is the wave number, Pr is the Prandtl number, $\mathrm{Br}$ is Brinkman number, $r$ is the radial coordinate and $\tau$ is the shear stress.

The constitutive equation for Casson's fluid in the non-dimensional form is given by (Casson (1959))

$$
\begin{aligned}
& \frac{\partial w}{\partial r}=-\left(\frac{1}{\mu(r)}\left[\sqrt{\tau_{r z}}-\sqrt{\tau_{0}}\right)^{2}, \tau_{r z} \geq \tau_{0}\right. \\
& \frac{\partial w}{\partial r}=0, \quad \tau_{r z} \leq \tau_{0}
\end{aligned}
$$


Incorporating Eq. (8) in Eqs. (5)-(7), and by using long wavelength and small Reynolds number approximation, we obtain

$$
\begin{aligned}
& \frac{1}{r} \frac{\partial}{\partial r}\left[r\left(\mu^{\frac{1}{2}}\left[\frac{\partial w}{\partial r}\right]^{\frac{1}{2}}\right)^{2}\right]=-\frac{\partial p}{\partial z}+\frac{\sin \beta}{F_{1}} \\
& 0=\frac{\partial p}{\partial r} \\
& \frac{1}{r}\left(r k(\theta) \frac{\partial \theta}{\partial r}\right)=B r\left(-\frac{\partial w}{\partial r}\left[\mu^{\frac{1}{2}}\left(\frac{\partial w}{\partial r}\right)^{\frac{1}{2}}+\tau_{0}^{\frac{1}{2}}\right]^{2}\right)
\end{aligned}
$$

The corresponding non-dimensional slip and convective boundary conditions are (Hayat et al. (2014))

$$
\begin{aligned}
& h \frac{\partial w}{\partial r}=-\frac{\alpha w}{\sqrt{D a}}, \frac{\partial \theta}{\partial r}+B i \theta=0 \text { at } r=h \\
& \frac{\partial \theta}{\partial r}=0, \tau_{r z} \text { is finite at } r=0
\end{aligned}
$$

where $\alpha$ is the velocity slip parameter, $D a$ is the porous parameter and $B i$ is the Biot number.

\section{SOLUTION OF THE PROBLEM}

The closed form solutions for the velocity in the core region is obtained by solving Eqs. (10) and (11) with the boundary conditions (13) and (14). We obtain the velocity expression as

$$
\begin{aligned}
w= & \frac{P+f}{2}\left[\frac { 1 } { \alpha _ { 1 } ^ { 2 } } \left(\alpha_{1}(r-h)-4 \alpha_{1}\left(\sqrt{r r_{p}}-\sqrt{h r_{p}}\right)+4 \sqrt{\alpha_{1} r_{p}}\right.\right. \\
& \left.\left(\tanh ^{-1} \sqrt{\alpha_{1} r}-\tanh ^{-1} \sqrt{\alpha_{1} h}\right)+\left(1+\alpha_{1} r_{p}\right) \log \frac{1-\alpha_{1} r}{1-\alpha_{1} h}\right) \\
& \left.+\frac{h \sqrt{D a}}{\alpha\left(1-\alpha_{1} h\right)}\left(h+r_{p}-\sqrt{h r_{p}}\right)\right]
\end{aligned}
$$

where $P=-\frac{\partial p}{\partial z}$ and $f=\frac{\sin \beta}{F_{1}}$.

Using the condition $\tau_{0}=\frac{P r_{p}}{2}$ at $r=r_{p}$, the upper limit of plug flow region is obtained as $r_{p}=\frac{2 \tau_{0}}{P}$. Also, by using the condition $\tau_{r z}=\tau_{h}$ at $r=h$ (Bird et al. (1976)), we obtain

$$
P=\frac{2 \tau_{h}}{h}
$$

Hence,

$$
\frac{r_{p}}{h}=\frac{\tau_{0}}{\tau_{h}}=\tau
$$

Using relation (17) in Eq. (15), we obtain the plug flow velocity as

$$
\begin{aligned}
w_{p}= & \frac{P+f}{2}\left[\frac { 1 } { \alpha _ { 1 } ^ { 2 } } \left(\alpha_{1}\left(r_{p}-h\right)-4 \alpha_{1}\left(r_{p}-\sqrt{h r_{p}}\right)+4 \sqrt{\alpha_{1} r_{p}}\right.\right. \\
& \left(\tanh ^{-1} \sqrt{\alpha_{1} r_{p}}-\tanh ^{-1} \sqrt{\alpha_{1} h}\right)+\left(1+\alpha_{1} r_{p}\right) \\
& \left.\left.\log \left(\frac{1-\alpha_{1} r_{p}}{1-\alpha_{1} h}\right)\right)+\frac{h \sqrt{D a}}{\alpha\left(1-\alpha_{1} h\right)}\left(h+r_{p}-\sqrt{h r_{p}}\right)\right]
\end{aligned}
$$

Integrating Eqs. (15) and (18) and using the conditions $\psi_{p}=0$ at $r=0$ and $\psi=\frac{q}{2}$ at $r=h$, the stream function for $r_{p} \leq r \leq h$ is given by

$$
\begin{array}{r}
\psi=\frac{q}{2}-\frac{P+f}{2}\left[G_{1}+G_{2}-G_{3}-G_{4}-G_{5}-G_{6}-G_{7}\right. \\
\left.-G_{8}+G_{9}-G_{10}-G_{4}\right]
\end{array}
$$

and stream function for plug flow in the region $0 \leq r \leq r_{p}$ can be written as

$$
\begin{aligned}
\psi_{p}= & \frac{(P+f) r^{2}}{4}\left[\frac { 1 } { \alpha _ { 1 } ^ { 2 } } \left(\alpha_{1}\left(r_{p}-h\right)-4 \alpha_{1}\left(r_{p}-\sqrt{h r_{p}}\right)+\right.\right. \\
& 4 \sqrt{\alpha_{1} r_{p}}\left(\tanh ^{-1} \sqrt{\alpha_{1} r_{p}}-\tanh ^{-1} \sqrt{\alpha_{1} h}\right)+\left(1+\alpha_{1} r_{p}\right) \\
& \left.\left.\log \left(\frac{1-\alpha_{1} r_{p}}{1-\alpha_{1} h}\right)\right)+\frac{h \sqrt{D a}}{\alpha\left(1-\alpha_{1} h\right)}\left(h+r_{p}-\sqrt{h r_{p}}\right)\right]
\end{aligned}
$$

The instantaneous volumetric flow rate in the wave frame is given by

$$
\begin{aligned}
q= & 2\left[\int_{0}^{r_{p}} w_{p} r d r+\int_{r_{p}}^{h} w r d r\right] \\
q= & \frac{P+f}{60 \alpha}\left[G_{12}\left(1+\alpha_{1} h\right)+\frac{G_{13}}{\alpha_{1}^{4}}+\frac{G_{14}}{\alpha_{1}^{3}}+\frac{G_{15}}{\alpha_{1}^{\frac{7}{2}}}+\frac{G_{16}}{\alpha_{1}^{2}}\right. \\
& \left.+\frac{G_{17}}{\alpha_{1}}+G_{18}\right]^{-1}
\end{aligned}
$$

Thus, we have

$$
\begin{aligned}
\frac{\partial p}{\partial z}= & f-60 \alpha q\left[G_{12}\left(1+\alpha_{1} h\right)+\frac{G_{13}}{\alpha_{1}^{4}}+\frac{G_{14}}{\alpha_{1}^{3}}+\frac{G_{15}}{\alpha_{1}^{\frac{7}{2}}}+\frac{G_{16}}{\alpha_{1}^{2}}\right. \\
& \left.+\frac{G_{17}}{\alpha_{1}}+G_{18}\right]^{-1}
\end{aligned}
$$

The dimensionless time-averaged flux $(\bar{Q})$ across one wavelength is

$$
\bar{Q}=\int_{0}^{1} \int_{0}^{h} r(w-1) d r d z=q+\int_{0}^{1} h^{2} d z=q+1+\frac{\epsilon^{2}}{2}
$$

The non-dimensional expression for pressure rise $(\Delta P)$ across one wavelength is given as follows:

$$
\Delta P=\int_{0}^{1} \frac{\partial p}{\partial z} d z
$$

The presence of nonlinear terms in heat transfer Eq. (12) makes it highly complex and hence it is difficult to get an exact solution. However, in the larger part of the practical problems, the value of $\phi$ is small which enables us to utilize a perturbation procedure to solve the nonlinear equation.

\subsection{Perturbation Method}

In order to obtain the perturbed solution about the temperature Eq. (12), the following procedure is employed. Expansion of $\theta$ leads to

$$
\theta=\sum_{n=0}^{\infty} \phi^{n} \theta_{n}
$$

\subsubsection{Zeroth order system}

$$
\begin{aligned}
& \frac{1}{r}\left(r k\left(\theta_{0}\right) \frac{\partial \theta_{0}}{\partial r}\right)=B r\left(-\frac{\partial w}{\partial r}\left[\mu^{\frac{1}{2}}\left(\frac{\partial w}{\partial r}\right)^{\frac{1}{2}}+\tau_{0}^{\frac{1}{2}}\right]^{2}\right) \\
& \frac{\partial \theta_{0}}{\partial r}+B i \theta_{0}=0 \text { at } r=h \\
& \frac{\partial \theta_{0}}{\partial r}=0 \text { at } r=0
\end{aligned}
$$

\subsubsection{First order system}

$$
\begin{aligned}
& \frac{1}{r}\left(r k\left(\theta_{1}\right) \frac{\partial \theta_{1}}{\partial r}\right)=B r\left(-\frac{\partial w}{\partial r}\left[\mu^{\frac{1}{2}}\left(\frac{\partial w}{\partial r}\right)^{\frac{1}{2}}+\tau_{0}^{\frac{1}{2}}\right]^{2}\right) \\
& \frac{\partial \theta_{1}}{\partial r}+B i \theta_{1}=0 \text { at } r=h \\
& \frac{\partial \theta_{1}}{\partial r}=0 \text { at } r=0
\end{aligned}
$$

On solving zeroth and first order system, and neglecting the terms higher than $O(\phi)$, we obtain the solution for temperature. 


\section{RESULTS AND DISCUSSION}

The Eq (25) is numerically integrated by using Weddle's rule in MATLAB. This section illustrates the role of physiological quantities such as axial velocity $(w)$, pressure rise $(\Delta P)$, time averaged flow rate $(\bar{Q})$ and trapping phenomenon $(\psi)$ on the peristaltic transport of Casson fluid. On the other hand, the role of variable thermal conductivity $(\phi)$ and convective boundary conditions $(B i)$ on temperature $(\theta)$ play a significant role in understanding the heat transfer characteristics of biofluids. Hence the present section shows the effects of yield stress $(\tau)$, porous parameter $(D a)$, velocity slip parameter $(\alpha)$, angle of inclination $(\beta)$, amplitude ratio $(\epsilon)$, thermal conductivity $(\phi)$, Biot number $(B i)$ and Brinkmann number $(\mathrm{Br})$ on physiological quantities.

\subsection{Velocity profile}

The effects of pertinent parameters on velocity distribution in the presence and absence of variable viscosity are elucidated through Fig. 2. Velocity profiles exhibit a parabolic nature with a maximum velocity occurs at the center of the tube. Fig. 2a is sketched to show the variation of $\tau$ on velocity profile. Here velocity profile decreases for higher values of $\tau$. Also, the peak velocity is attained when $\tau=0$. This is because of the yield stress parameter present in the model requires some amount of energy to begin the flow and hence an increase in the value of this parameter results in a decrease in the velocity. Figs $2 b$ and $2 c$ are drawn to show the effects of $D a$ and $\alpha$ on velocity. Results indicate that the velocity is an increasing function of $D a$. However, the opposite behavior is noticed for higher values of $\alpha$. Fig. $2 d$ indicates that the velocity field enhances for an increase in the value of $\beta$. However, in all the cases, the velocity field enhance for a higher value of viscosity $\left(\alpha_{1}=0.1\right)$. Thus, the presence of variable viscosity plays a vital role in enhancing the velocity field in an inclined porous tube.

\subsection{Temperature profile}

The effects of $\alpha, \tau, D a, \phi, B i$ and $B r$ on temperature are plotted and discussed in the Figs. 3 and 4. Fig. 3a shows the variation of $\alpha_{1}$ on temperature. From the figure, it is clear that the temperature diminishes for higher values of variable viscosity. This decay in temperature subject to a decrease in $\alpha_{1}$ is uniform throughout the tube. Fig. 3b reveals that the enhancement in the magnitude of temperature is because of the higher values of $\tau$. Fig. 4a portrays the variation of $D a$ on temperature. Here the decay in temperature is observed near the axis of the tube, and opposite behavior is noticed near the walls of the tube. Fig. 4b is graphed to illustrate the effect of $\phi$ on temperature. An increment in $\phi$ results in an increase in the temperature near the axis of the tube and the effect is negligible as we move towards the wall. This is because higher values of $\phi$ allow the fluid to dissipate or absorb the heat to its surroundings. Thus, when the temperature of the fluid is lower than the temperature of the boundary, an increment in the value of $\phi$ enhances the temperature near the axis of the tube. Fig. $4 \mathrm{c}$ depicts the variation of temperature due to the influence of $B i$. An increase in the value of $B i$ results in the reduction of temperature. Fig. $4 \mathrm{~d}$ shows the effect of $B r$ on temperature. Here an increment in $\mathrm{Br}$ enhances the temperature. Because $\mathrm{Br}$ occurs due to the viscous dissipation effects and it enhances the temperature.

\subsection{Pumping characteristics}

Pressure rise per wavelength is analysed in three different regions which are pumping region $(\Delta P>0, \bar{Q}>0)$, free pumping region $(\Delta P=0)$ and augmented region $(\Delta P<0, \bar{Q}<0)$. The quantitative analysis for the effects of pertinent parameters on pumping performance in the intervals for $\bar{Q}$, when $\Delta P>0$ and $\Delta P<0$ are presented in Table 1 . We observe that an increase in the value of $D a$ and $\beta$ increases the length of the interval in the pumping region and opposite behavior is observed in the augmented region. This is mainly because an increase in the value of $D a$ increases the porosity of the wall and thus $\bar{Q}$ decreases. Also, for a fixed value of $D a$ and $\beta$, the length of the pumping region increases in the case of variable viscosity with that of constant viscosity. Further, the effects of $\tau, \alpha$, and $\epsilon$ decrease the length of the interval for $\Delta P>0$ and opposite behavior is observed when viscosity increases from 0 to 0.1 . This information on $\tau$ helps in equalizing the pumping rate of Newtonian and Casson fluid for some value of $\bar{Q}$ by adjusting the peristalsis velocity.

(a)

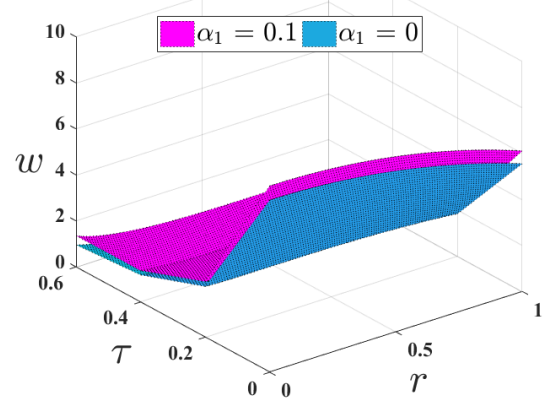

(b)

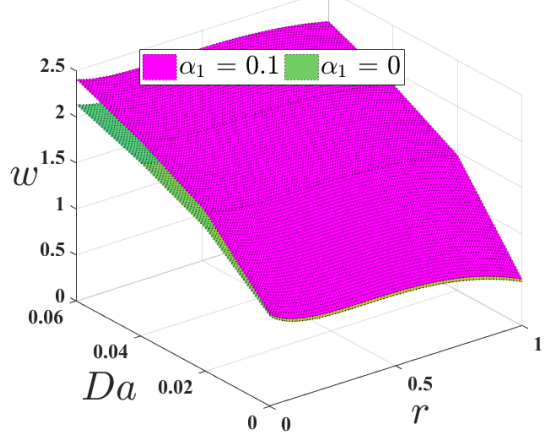

(c)

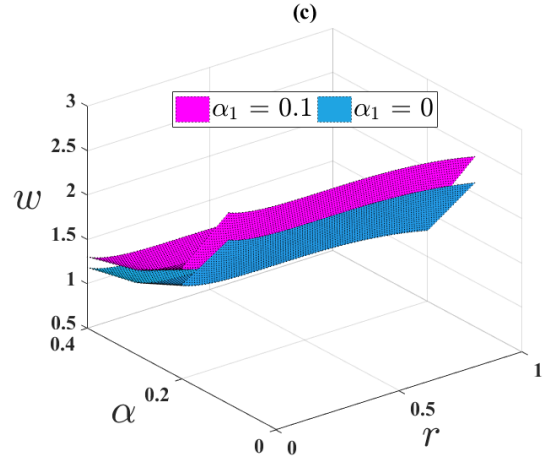

(d)

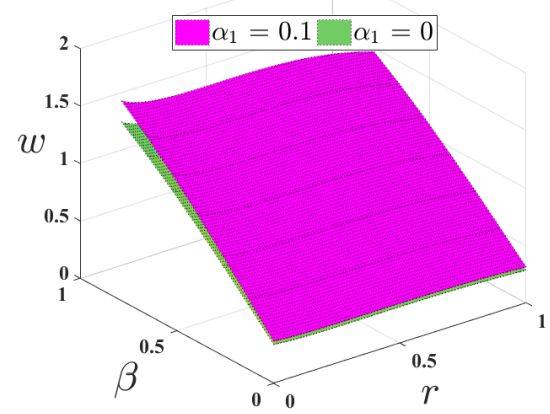

Fig. 2 Velocity profile for varying (a) yield stress, (b) porous parameter, (c) velocity slip parameter and (d) angle of inclination. 
(a)

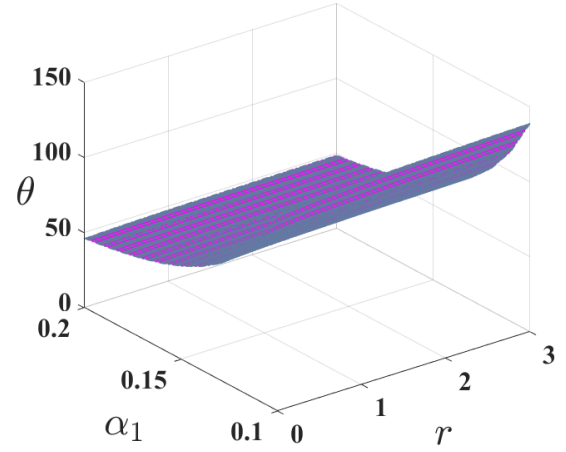

(b)

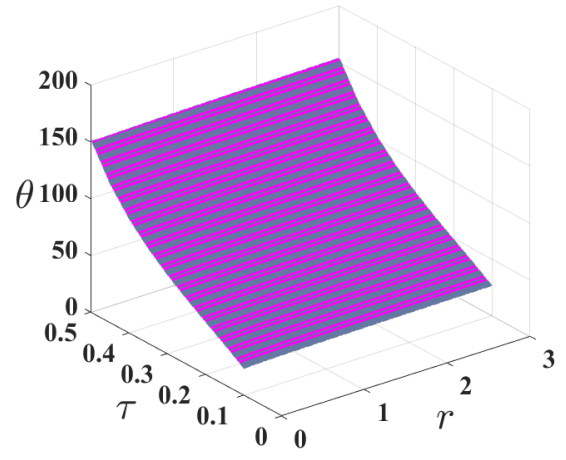

Fig. 3 Temperature profile for varying (a) variable viscosity and (b) yield stress

\subsection{Trapping Phenomenon}

The most fundamental part of peristalsis is trapping. It is the formation of the inside flowing bolus. This phenomenon is helpful in understanding the movement of the gastrointestinal tract and in the arrangement of thrombus in veins. Fig. 5 reveals that an increase in the value of $\alpha$ diminishes the size of the trapped bolus. Figs. 6 and 7 represents the impact of $\tau$ and $D a$ on the trapped bolus. An expansion in the estimation of $\tau$ and $D a$ enhances the volume of the trapped bolus.

\section{CONCLUSIONS}

The present investigation analyzes the impact of variable liquid properties on the peristaltic mechanism of Casson liquid in an inclined porous tube. The examination finds its application in understanding the complex rheological behavior of biological fluids. Specifically, the flow of blood in narrow arteries and the movement of chyme in the gastrointestinal tract. The future investigation can be made by including the magnetic effect to the present model so that it can give a better understanding of the flow of blood when exposed to an external magnetic field. The main findings from the current model are listed below:

- The axial velocity field is an increasing function of $D a, \epsilon$, and $\beta$ while it reduces for $\tau$ and $\alpha$.

- The magnitude of temperature decreases for higher value of $\alpha_{1}$ and $B i$, and it increases for an increase in the value of $\tau$.

- Temperature of the fluid enhances for a larger value of $\phi$.

- Pressure rise enhances for $\tau, \alpha, \beta$ and $\epsilon$, and it diminishes for $D a$.

- Variable viscosity plays a significant role in enhancing pressure rise.
- The size of trapped bolus increases for $\tau, D a$ and $\epsilon$, and it reduces for $\alpha$.

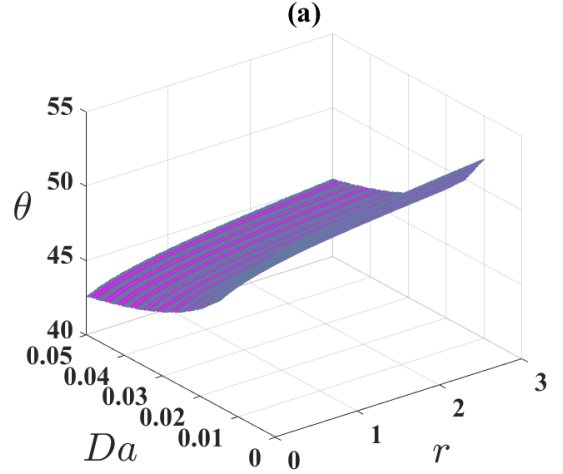

(b)

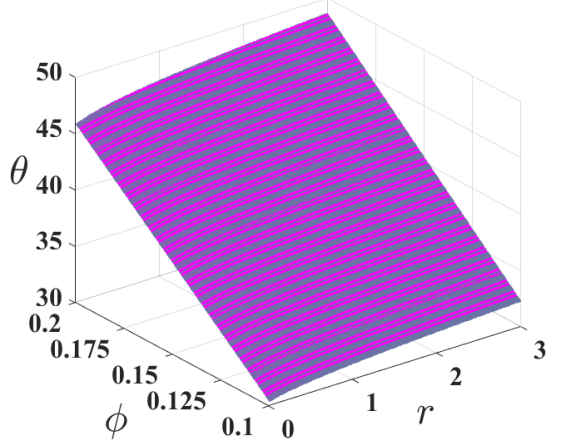

(c)

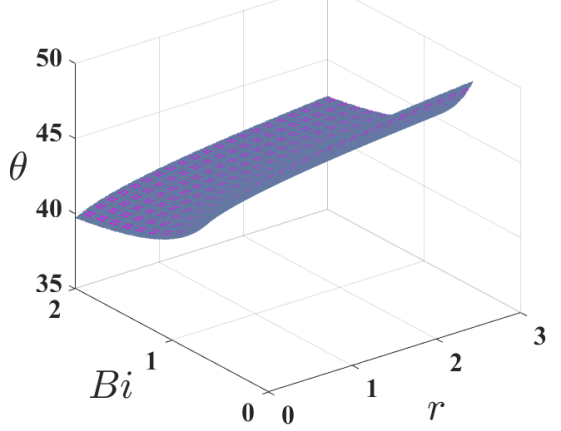

(d)

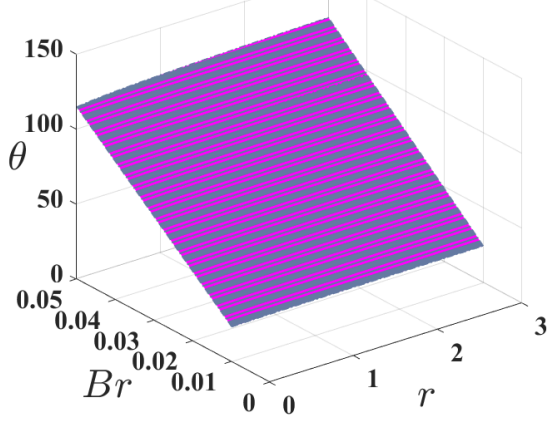

Fig. 4 Temperature profile for varying (a) porous parameter, (b) variable thermal conductivity, (c) Biot number and (d) Brinkmann number. 
(a)

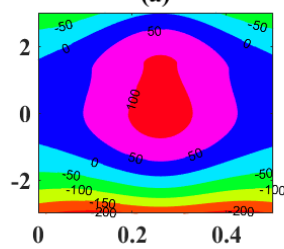

(c)

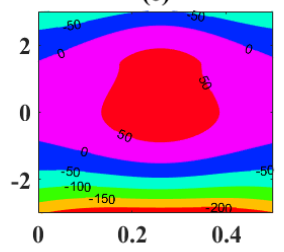

(b)

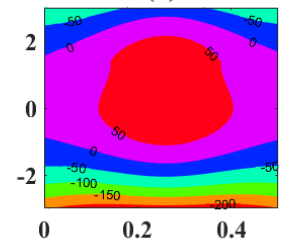

(d)

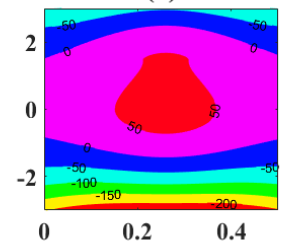

Fig. 5 Streamlines for varying (a) $\alpha=0.2$, (b) $\alpha=0.22$, (c) $\alpha=0.24$ and (d) $\alpha=0.26$.

(a)

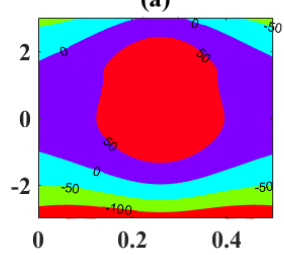

(c)

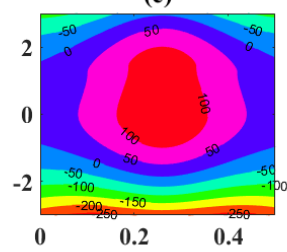

(b)

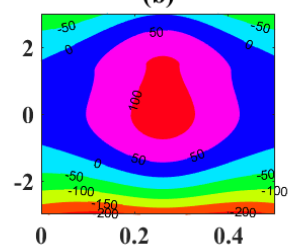

(d)

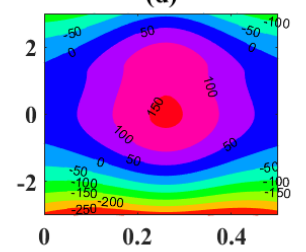

Fig. 6 Streamlines for varying (a) $\tau=0.1$, (b) $\tau=0.2$, (c) $\tau=0.3$ and (d) $\tau=0.4$.

(a)

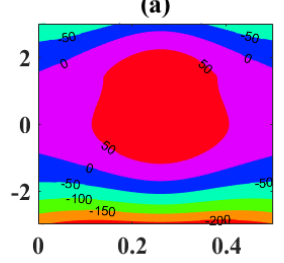

(c)

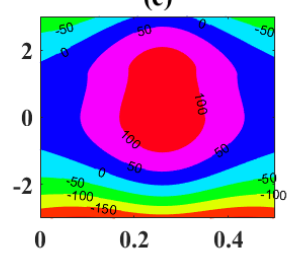

(b)

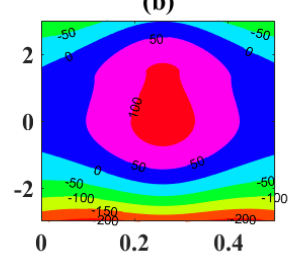

(d)

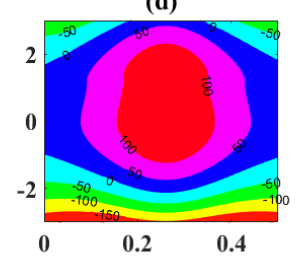

Fig. 7 Streamlines for varying (a) $D a=0.01$, (b) $D a=0.02$, (c) $D a=$ 0.03 and (d) $D a=0.04$.

\section{NOMENCLATURE}

$\begin{array}{ll}a & \text { radius of the tube } \\ b & \text { amplitude } \\ t & \text { time } \\ c & \text { wave speed } \\ p & \text { pressure } \\ P & \text { pressure gradient } \\ \operatorname{Pr} & \text { Prandtl number } \\ E c & \text { Eckert number } \\ B r & \text { Brinkmann number } \\ D a & \text { porous parameter (Darcy number) } \\ F_{1} & \text { body force parameter } \\ B i & \text { Biot number } \\ r, z & \text { radial and axial coordinates } \\ u, w & \text { velocity in radial and axial directions } \\ q & \text { volumetric flow rate } \\ \operatorname{Re} & \text { Reynolda number } \\ \bar{Q} & \text { time averaged volumetric flow rate } \\ \Delta P & \text { Pressure rise } \\ c_{p} & \text { specific heat at constant pressure } \\ k & \text { thermal conductivity } \\ g & \text { acceleratio due to gravity } \\ G r e e k & \text { Symbols } \\ \alpha & \text { velocity slip parameter } \\ \alpha & \text { coefficient of variable viscosity } \\ \beta & \text { angle of incliation } \\ \phi & \text { coefficient of variable thermal conductivity } \\ \lambda & \text { wavelength } \\ \tau & \text { ratio of yield stress to wall shearing stress } \\ \tau_{0} & \text { yield stress } \\ \mu & \text { viscosity of the fluid } \\ \mu_{0} & \text { constant viscosity } \\ \epsilon & \text { amplitude ratio } \\ \psi & \text { stream function } \\ \theta & \text { temperature } \\ \delta & \text { wave number } \\ \rho & \text { density } \\ & \end{array}$

\section{REFERENCES}

Akbar, N.S., 2014, "Double-difusive natural convective peristaltic flow of a Jeffery nanofluid in a porous channel," Heat Transfer Research, 45(4), 293-307.

https://doi.org/10.1615/HeatTransRes.2013006995 .

Bhatti, M.M., and Zeeshan, A., 2016, "Analytic study of heat transfer with variable viscosity on solid particle motion in dusty Jeffery fluid," Modern Physics Letters B, 30(16), 1650196. https://doi.org/10.1142/S0217984916501967.

Bird, R.B., Stewart, W.E., and Lightfoot, E.N., 1976, Transport Phenomena, Wiley, Newyork.

Casson, N., 1959, Rheology of Disperse systems, Pergamon Press, New York.

Hayat, T., F, M.A., Ahmad, B., and Alsaedi, A., 2014, "MHD Mixed Convection Peristaltic Flow with Variable Viscosity and Thermal Conductivity," Sains Malaysiana, 43(10), 1583-1590.

Hayat, T., Sadaf, N., Alsaedi, A., and Rafiq, M., 2016, "Impact of secondorder velocity and thermal slips in the mixed convective peristalsis with carbon nanotubes and porous medium," Journal of Molecular Liquids, 221, 434-442.

https://doi.org/10.1016/j.molliq.2016.05.072. 
Table 1 Interval for time averaged flow rate $\bar{Q}$ across one wavelength for different values of the physiological parameters when $F_{1}=0.1$ and $t=0.01$.

\begin{tabular}{|ccccc|c|c|c|c|}
\hline \multicolumn{6}{|c|}{ Parameters } & \multicolumn{2}{c|}{$\alpha_{1}=0$} & \multicolumn{2}{c|}{$\alpha_{1}=0$} \\
\hline$\epsilon$ & $\tau$ & $\beta$ & $\alpha$ & $D a$ & $\Delta P>0$ & $\Delta P<0$ & $\Delta P>0$ & $\Delta P<0$ \\
\hline 0.5 & 0.2 & $\frac{\pi}{4}$ & 0.2 & 0 & $0<\bar{Q}<1.182$ & $1.182<\bar{Q}<2$ & $0<\bar{Q}<1.185$ & $1.185<\bar{Q}<2$ \\
& & & & 0.01 & $0<\bar{Q}<1.312$ & $1.312<\bar{Q}<2$ & $0<\bar{Q}<1.338$ & $1.338<\bar{Q}<2$ \\
& & & & 0.015 & $0<\bar{Q}<1.342$ & $1.342<\bar{Q}<2$ & $0<\bar{Q}<1.372$ & $1.372<\bar{Q}<2$ \\
& & & & 0.02 & $0<\bar{Q}<1.366$ & $1.366<\bar{Q}<2$ & $0<\bar{Q}<1.401$ & $1.401<\bar{Q}<2$ \\
\hline 0.5 & 0.2 & $\frac{\pi}{4}$ & 0.1 & 0.02 & $0<\bar{Q}<1.551$ & $1.551<\bar{Q}<2$ & $0<\bar{Q}<1.618$ & $1.618<\bar{Q}<2$ \\
& & & 0.2 & & $0<\bar{Q}<1.366$ & $1.366<\bar{Q}<2$ & $0<\bar{Q}<1.401$ & $1.401<\bar{Q}<2$ \\
& & & 0.3 & & $0<\bar{Q}<1.305$ & $1.305<\bar{Q}<2$ & $0<\bar{Q}<1.329$ & $1.329<\bar{Q}<2$ \\
& & & 0.4 & & $0<\bar{Q}<1.274$ & $1.274<\bar{Q}<2$ & $0<\bar{Q}<1.293$ & $1.293<\bar{Q}<2$ \\
\hline 0.5 & 0.2 & 0 & 0.2 & 0.02 & $0<\bar{Q}<1.125$ & $1.125<\bar{Q}<2$ & $0<\bar{Q}<1.125$ & $1.125<\bar{Q}<2$ \\
& & $\frac{\pi}{12}$ & & & $0<\bar{Q}<1.213$ & $1.213<\bar{Q}<2$ & $0<\bar{Q}<1.226$ & $1.226<\bar{Q}<2$ \\
& & $\frac{\pi}{8}$ & & & $0<\bar{Q}<1.255$ & $1.255<\bar{Q}<2$ & $0<\bar{Q}<1.274$ & $1.274<\bar{Q}<2$ \\
& & $\frac{\pi}{4}$ & & & $0<\bar{Q}<1.366$ & $1.366<\bar{Q}<2$ & $0<\bar{Q}<1.401$ & $1.401<\bar{Q}<2$ \\
\hline 0.5 & 0.2 & $\frac{\pi}{4}$ & 0.2 & 0.02 & $0<\bar{Q}<1.366$ & $1.366<\bar{Q}<2$ & $0<\bar{Q}<1.401$ & $1.401<\bar{Q}<2$ \\
& 0.4 & & & & $0<\bar{Q}<1.267$ & $1.267<\bar{Q}<2$ & $0<\bar{Q}<1.242$ & $1.242<\bar{Q}<2$ \\
& 0.6 & & & & $0<\bar{Q}<1.205$ & $1.205<\bar{Q}<2$ & $0<\bar{Q}<1.166$ & $1.166<\bar{Q}<2$ \\
\hline 0.4 & 0.2 & $\frac{\pi}{4}$ & 0.2 & 0.02 & $0<\bar{Q}<1.477$ & $1.477<\bar{Q}<2$ & $0<\bar{Q}<1.541$ & $1.541<\bar{Q}<2$ \\
0.5 & & & & & $0<\bar{Q}<1.366$ & $1.366<\bar{Q}<2$ & $0<\bar{Q}<1.401$ & $1.401<\bar{Q}<2$ \\
0.6 & & & & & $0<\bar{Q}<1.304$ & $1.304<\bar{Q}<2$ & $0<\bar{Q}<1.321$ & $1.321<\bar{Q}<2$ \\
\hline
\end{tabular}

Hayat, T., Ullah, I., Alsaedi, A., and Ahmad, B., 2018a, "Impact of temperature dependent heat source and nonlinear radiative flow of third grade fluid with chemical aspects," Thermal Science.

https://doi.org/10.2298/TSCI180409245H.

Hayat, T., Ullah, I., Alsaedi, A., and Asghar, S., 2018b, "Flow of magneto Williamson nanoliquid towards stretching sheet with variable thickness and double stratification," Radiation Physics and Chemistry, 152, 151157.

https://doi.org/10.1016/j.radphyschem.2018.07.006.

Hayat, T., Ullah, I., Alsaedi, A., and Asghar, S., 2018c, "Magnetohydrodynamics Stagnation-Point Flow of Sisko Liquid With Melting Heat Transfer and Heat Generation/Absorption," Journal of Thermal Science and Engineering Applications, 10(5), 051015.

https://doi.org/10.1115/1.4040032.

Hayat, T., and Ali, N., 2008, "Effect of variable viscosity on the peristaltic transport of a Newtonian fluid in an asymmetric channel," Applied Mathematical Modelling, 32(5), 761-774.

https://doi.org/10.1016/j.apm.2007.02.010.

Hussain, Q., Asghar, S., Hayat, T., and Alsaedi, A., 2015, "Heat transfer analysis in peristaltic flow of MHD Jeffrey fluid with variable thermal conductivity," Applied Mathematics and Mechanics, 36(4), 499-516. https://doi.org/10.1007/s10483-015-1926-9.

Khalid, A., Khan, I., Khan, A., and Shafie, S., 2015, "Unsteady MHD free convection flow of Casson fluid past over an oscillating vertical plate embedded in a porous medium," Engineering Science and Technology, an International Journal, 18(3), 309-317.

https://doi.org/10.1016/j.jestch.2014.12.006.

Khan, K.A., Butt, A.R., and Raza, N., 2018, "Effects of heat and mass transfer on unsteady boundary layer flow of a chemical reacting Casson fluid," Results in Physics, 8, 610 - 620 .

https://doi.org/10.1016/j.rinp.2017.12.080.

Kumar, S.R., and Abzal, S.K., 2017, "Combined influence of Hall currents and Joule heating on hemodynamic peristaltic flow with porous medium through a vertical tapered asymmetric channel with radiation,"
Frontiers in Heat and Mass Transfer, 9, 19. https://doi.org/10.5098/hmt.9.19.

Manjunatha, G., and Rajashekhar, C., 2018, "Slip effects on peristaltic transport of Casson fluid in an inclined elastic tube with porous walls," Journal of Advanced Fluid Mechanics and Thermal Sciences, 3(1), 6780 .

Mebarek-Oudina, F., and Bessaih, R., 2016, "Oscillatory Magnetohydrodynamic Natural Convection of Liquid Metal between Vertical Coaxial Cylinders," Journal of Applied Fluid Mechanics, 9(4), 1655-1665.

Mernone, A., Mazumdar, J., and Lucas, S., 2002, "A mathematical study of peristaltic transport of a casson fluid," Mathematical and Computer Modellin, 35(7), 895-912.

https://doi.org/10.1016/S0895-7177(02)00058-4.

Nadeem, S., and Akbar, N.S., 2009, "Influence of heat transfer on a peristaltic transport of Herschel-Bulkley fluid in a non-uniform inclined tube," Communications in Nonlinear Science and Numerical Simulation, 14(12), 4100-4113.

https://doi.org/10.1016/j.cnsns.2009.02.032.

Prasad, K.V., Vaidya, H., Vajravelu, K., and Rashidi, M.M., 2017a, "Effects of Variable Fluid Properties on MHD Flow and Heat Transfer over a Stretching Sheet with Variable Thickness,' Journal of Mechanics, 33(4), 501-512.

https://doi.org/10.1017/jmech.2016.101.

Prasad, K., Vajravelu, K., Vaidya, H., Basha, N.Z., and Umesh, V., 2017b, "Thermal and species concentration of MHD Casson fluid at a vertical sheet in the presence variable fluid properties," Ain Shams Engineering Journal.

https://doi.org/10.1016/j.asej.2016.08.017.

Rajashekhar, C., Manjunatha, G., Prasad, K.V., Divya, B.B., and Vaidya, H., 2018, "Peristaltic transport of two-layered blood flow using HerschelBulkley Model," Cogent Engineering, 5(1), 1495592.

https://doi.org/10.1080/23311916.2018.1495592. 
Raju, K.K., and Devanathan, R., 1972, "Peristaltic motion of a nonNewtonian fluid," Rheologica Acta, 11(2), 170-178.

https://doi.org/10.1007/BF01993016.

Ramesh, K., 2016, "Effects of slip and convective conditions on the peristaltic flow of couple stress fluid in an asymmetric channel through porous medium," Computer Methods and Programs in Biomedicine, 135, $1-14$.

https://doi.org/10.1016/j.cmpb.2016.07.001.

Ramesh, K., and Devakar, M., 2015, "Some analytical solutions for flows of Casson fluid with slip boundary conditions," Ain Shams Engineering Journal, 6(3), 967-975.

https://doi.org/10.1016/j.asej.2015.02.007.

Sankad, G.C., and Patil, A., 2018, "Heat transfer influences on the Herschel-Bulkley fluid flow under peristalsis," Frontiers in Heat and Mass Transfe, 10, 17.

https://doi.org/10.5098/hmt.10.17 .

Shapiro, A.H., Jaffrin, M.Y., and Weinberg, S.L., 1969, "Peristaltic pumping with long wavelengths at low Reynolds number," Journal of Fluid Mechanics, 37(4), 799-825.

https://doi.org/10.1017/S0022112069000899.

Sinha, A., Shit, G., and Ranjit, N., 2015, "Peristaltic transport of MHD flow and heat transfer in an asymmetric channel: Effects of variable viscosity, velocity-slip and temperature jump," Alexandria Engineering Journa, 54(3), 691-704.

https://doi.org/10.1016/j.aej.2015.03.030.

Srivastava, L.M., and Srivastava, V.P., 1984, "Peristaltic transport of blood: Casson model-II," Journal of Biomechanics, 17(11), 821-829. https://doi.org/10.1016/0021-9290(84)90140-4.

Vaidya, H., Manjunatha, G., Rajashekhar, C., and Prasad, K.V., 2018, "Role of slip and heat transfer on peristaltic transport of Herschel-Bulkley fluid thorugh an elastic tube," Multidiscipline Modelling in Materials and Structures.

https://doi.org/10.1108/MMMS-11-2017-0144.

Vajravelu, K., Prasad, K.V., Vaidya, H., Basha, N.Z., and Chiu-On, N., 2017, "Mixed Convective Flow of a Casson Fluid over a Vertical Stretching Sheet," International Journal of Applied and Computational Mathematics, 3(3), 1619-1638.

https://doi.org/10.1007/s40819-016-0203-6.

Vajravelu, K., Sreenadh, S., and Babu, V.R., 2005, "Peristaltic transport of a Herschel-Bulkley fluid in an inclined tube," International Journal of Non-Linear Mechanics, 40(1), 83-90.

https://doi.org/10.1016/j.ijnonlinmec.2004.07.001.

Wakif, A., Boulahia, Z., Farhad, A., Eid, M.R., and Sehaqui, R., 2018a, "Numerical Analysis of the Unsteady Natural Convection MHD Couette Nanofluid Flow in the Presence of Thermal Radiation Using Single and Two-Phase Nanofluid Models for $\mathrm{Cu}$-Water Nanofluids," International Journal of Applied and Computational Mathematics, 4(3), 81. https://doi.org/10.1007/s40819-018-0513-y.

Wakif, A., Boulahia, Z., Mishra, S.R., Rashidi, M.M., and Sehaqui, R., 2018b, "Influence of a uniform transverse magnetic field on the thermohydrodynamic stability in water-based nanofluids with metallic nanoparticles using the generalized Buongiorno's mathematical mode," The European Physical Journal Plus, 133(5), 181. https://doi.org/10.1140/epjp/i2018-12037-7.
Wakif, A., Boulahia, Z., and Sehaqui, R., 2018c, "A semi-analytical analysis of electro-thermo-hydrodynamic stability in dielectric nanofluids using Buongiorno's mathematical model together with more realistic boundary conditions," Results in Physics, 9, 1438-1454. https://doi.org/10.1016/j.rinp.2018.01.066.

Zia, Q.M.Z., Ullah, I., Waqas, M., Alsaedi, A., and Hayat, T., 2018, "Cross diffusion and exponential space dependent heat source impacts in radiated three-dimensional (3D) flow of Casson fluid by heated surface," Results in Physics, 8, 1275 - 1282. https://doi.org/10.1016/j.rinp.2018.01.001.

\section{APPENDIX}

The expressions that appear in sections 2 and 3 are listed as follows

$$
\begin{aligned}
& G_{1}=\frac{h^{3}}{3 \alpha_{1}}+\frac{2 \sqrt{\alpha_{1} h}\left(3+\alpha_{1} h\right) \sqrt{\alpha_{1} r_{p}}}{3 \alpha_{1}^{4}}, \\
& G_{2}=\frac{\sqrt{D a} h^{3} \sqrt{r_{p}}}{\alpha\left(\alpha+\alpha_{1} h\right)}-\frac{h+\alpha_{1} h r_{p}}{2 \alpha_{1}^{3}}, \\
& G_{3}=\frac{h^{2}\left(2 \alpha_{1}^{2} \sqrt{D a} h\left(h+r_{p}\right)+\alpha\left(-1+\alpha_{1} h\right)\left(1+\alpha_{1}^{2}\left(2 h+r_{p}\right)\right)\right)}{4 \alpha \alpha_{1}^{2}\left(-1+\alpha_{1} h\right)}, \\
& G_{4}=\frac{2 \sqrt{r_{p}} \tanh ^{-1} \sqrt{\alpha_{1} h}}{\alpha_{1}^{\frac{7}{2}}}, G_{5}=\frac{\left(1+\alpha_{1} r_{p}\right) \log \left(1-\alpha_{1} h\right)}{2 \alpha_{1}^{4}}, \\
& G_{6}=\frac{r^{3}}{3 \alpha_{1}}+\frac{2 \sqrt{\alpha_{1} r}\left(3+\alpha_{1} r\right) \sqrt{\alpha_{1} r_{p}}}{3 \alpha_{1}^{4}}, \\
& G_{7}=\frac{\sqrt{D a} h r^{2} \sqrt{h r_{p}}}{\alpha\left(-1+\alpha_{1} h\right)}+\frac{8(h-r)(2 h+3 r) \sqrt{(r-h) r_{p}}}{15 \alpha_{1}}, \\
& G_{8}=\frac{r+\alpha_{1} r r_{p}}{2 \alpha_{1}^{3}}+\frac{r^{2}\left(2 \alpha_{1}^{2} \sqrt{D a} h\left(h+r_{p}\right)+\alpha\left(-1+\alpha_{1} h\right)\left(1+\alpha_{1}^{2}\left(2 h+r_{p}\right)\right)\right)}{4 \alpha \alpha_{1}^{2}\left(-1+\alpha_{1} h\right)} \text {, } \\
& G_{9}=\frac{2 r^{2} \sqrt{\alpha_{1} r_{p}} t a h^{-1} \sqrt{\alpha_{1} h}}{\alpha_{1}^{2}}+\frac{2 \sqrt{r_{p}} \tanh ^{-1} \sqrt{\alpha_{1} r}}{\alpha_{1}^{\frac{7}{2}}}, \\
& G_{10}=\frac{2 r^{2} \sqrt{\alpha_{1} r_{p}} \tanh ^{-1} \sqrt{\alpha_{1} r}}{\alpha_{1}^{2}}+\frac{r^{2}\left(1+\alpha_{1} r_{p}\right) \log \left(\frac{-1+\alpha_{1} r}{-1+\alpha_{1} h}\right)}{2 \alpha_{1}^{2}}, \\
& G_{12}=30 \sqrt{D a} h^{3}(h+\tau h-2 h \sqrt{2}), G_{13}=30 \alpha \log \left(\frac{1-\alpha_{1} \tau h}{1-\alpha_{1} h}\right), \\
& G_{14}=30 \alpha h\left[\sqrt{\tau}(4-3 \sqrt{\tau})+\tau \log \left(\frac{1-\alpha_{1} \tau h}{1-\alpha_{1} h}\right)-1\right], \\
& G_{15}=120 \alpha \sqrt{\tau h}\left(\tanh ^{-1} \sqrt{\alpha_{1} \tau h}-\tanh ^{-1} \sqrt{\alpha_{1} h}\right), \\
& G_{16}=\alpha h\left[10 h \sqrt{\tau}(4-3 \sqrt{\tau})+5 h r^{2}\left(1+6 \log \left(\frac{1-\alpha_{1} h}{1-\alpha_{1} \tau h}\right)\right)-15 h\right], \\
& G_{17}=\alpha h\left[3 h^{2} \sqrt{\tau}(8-5 \sqrt{\tau})+h^{2} r^{3}\left(1+30 \log \left(\frac{1-\alpha_{1} h}{1-\alpha_{1} \tau h}\right)\right)-10 h^{2}\right] \text {, } \\
& G_{18}=\frac{30 \alpha h^{2} \tau^{2}}{\alpha_{1}} \log \left(\frac{\alpha_{1} \tau h-1}{\alpha_{1} h-1}\right)\left(\frac{1}{\alpha_{1}}+\tau h\right), \\
& G_{19}=9 \alpha_{1} r\left(8+\alpha_{1} r\right)-32 \alpha_{1} \sqrt{r}\left(18+\alpha_{1} r\right) \sqrt{r_{p}}, \\
& G_{20}=36 \alpha_{1}^{2} r r_{p}-144 \sqrt{\alpha_{1} r_{p}} \tanh ^{-1} \sqrt{\alpha_{1} r}, G_{21}=\log \left(\frac{1-\sqrt{\alpha_{1} r}}{1+\sqrt{\alpha_{1} r}}\right) \text {, } \\
& G_{22}=36 \log (r)+36 \alpha_{1} r_{p} \log (r)-720 \sqrt{\alpha_{1} r_{p}} \operatorname{polylog}\left(2, \sqrt{\alpha_{1} h}\right) \text {, } \\
& G_{23}=8 \sqrt{h}\left(-45+2 h\left(-36 B i+\alpha_{1}\left(15+34 B i h+\alpha_{1} h\right.\right.\right. \\
& (3+2 B i h)))) \sqrt{r_{p}}, G_{25}=-36\left(-1+\alpha_{1} h\left(-1+\alpha_{1} h\right)(1+B i h)\right) r_{p} \text {, } \\
& G_{24}=-9 h\left(\alpha_{1} h-1\right)\left(8+8 B i h+\alpha_{1} h(2+B i h)\right), \\
& G_{26}=2 \sqrt{\alpha_{1}}(-5+2 B i h) \sqrt{r_{p}} \tanh ^{-1} \sqrt{\alpha_{1} h}, \\
& G_{27}=\log \left(1+\sqrt{\alpha_{1} h}\right)-\log \left(1-\sqrt{\alpha_{1} h}\right)-\log (h), \\
& G_{28}=108 \log \left(1-\alpha_{1} h\right)-36 \alpha_{1}\left(-2 r_{p}+h\left(3+2 \alpha_{1} r_{p}\right)\right) \log \left(1-\alpha_{1} h\right) \text {, } \\
& G_{29}=36 \operatorname{Bih}\left(\alpha_{1} h-1\right)\left(5+5 \sqrt{\alpha_{1} r_{p}}+2 \alpha_{1} r_{p}\right) \operatorname{polylog}\left(2, \alpha_{1} h\right), \\
& G_{30}=720 \sqrt{\alpha_{1} r_{p}} \operatorname{polylog}\left(2, \sqrt{\alpha_{1} r}\right)-36\left(3+5 \sqrt{\alpha_{1} r_{p}}+2 \alpha_{1} r_{p}\right) \\
& \text { polylog }\left(2, \alpha_{1} r\right) \text {. }
\end{aligned}
$$

